Article

\title{
Steady-State Levels of Cytokinins and Their Derivatives May Serve as a Unique Classifier of Arabidopsis Ecotypes
}

\author{
Zuzana Samsonová ${ }^{1}$, Nagavalli S. Kiran ${ }^{1}$, Ondřej Novák ${ }^{2}{ }^{\circledR}$, Ioannis Spyroglou ${ }^{3}{ }^{\circledR}$, Jan Skalák ${ }^{4}$, \\ Jan Hejátko ${ }^{4}\left(\mathbb{D}\right.$ and Vít Gloser ${ }^{1, *}$ \\ 1 Department of Experimental Biology, Faculty of Science, Masaryk University Kamenice 5, Brno 62500, \\ Czech Republic; zuzka.samsonova@gmail.com (Z.S.); naasuki@gmail.com (N.S.K.) \\ 2 Faculty of Science, Palacký University and Laboratory of Growth Regulators, Institute of Experimental \\ Botany, The Czech Academy of Sciences, Šlechtitelů 27, Olomouc 78371, Czech Republic; \\ ondrej.novak@upol.cz \\ 3 Plant Sciences Core Facility, CEITEC MU, Central European Institute of Technology, Masaryk University, \\ Kamenice 5, Brno 62500, Czech Republic; ioannis.spyroglou@ceitec.muni.cz \\ 4 Functional Genomics \& Proteomics of Plants, CEITEC MU, Central European Institute of Technology, \\ Kamenice 5, Brno 62500, Czech Republic; jan.skalak@ceitec.muni.cz (J.S.); hejatko@sci.muni.cz (J.H.) \\ * Correspondence: vitgloser@sci.muni.cz
}

Received: 10 December 2019; Accepted: 15 January 2020; Published: 17 January 2020

\begin{abstract}
We determined steady-state (basal) endogenous levels of three plant hormones (abscisic acid, cytokinins and indole-3-acetic acid) in a collection of thirty different ecotypes of Arabidopsis that represent a broad genetic variability within this species. Hormone contents were analysed separately in plant shoots and roots after 21 days of cultivation on agar plates in a climate-controlled chamber. Using advanced statistical and machine learning methods, we tested if basal hormonal levels can be considered a unique ecotype-specific classifier. We also explored possible relationships between hormone levels and the prevalent environmental conditions in the site of origin for each ecotype. We found significant variations in basal hormonal levels and their ratios in both root and shoot among the ecotypes. We showed the prominent position of cytokinins (CK) among the other hormones. We found the content of CK and CK metabolites to be a reliable ecotype-specific identifier. Correlation with the mean temperature at the site of origin and the large variation in basal hormonal levels suggest that the high variability may potentially be in response to environmental factors. This study provides a starting point for ecotype-specific genetic maps of the CK metabolic and signalling network to explore its contribution to the adaptation of plants to local environmental conditions.
\end{abstract}

Keywords: abscisic acid; cytokinins; cytokinin glucosides; cytokinin metabolism; indole-3-acetic acid; single nucleotide polymorphism

\section{Introduction}

Plant evolution has been shaped by many factors including environmental conditions or the size and geographical distribution of populations of individual species. It has been shown that large variability in morphology and functions may exist even among populations of the same species. An increased incidence of single nucleotide polymorphisms (SNPs) has been reported among genes mediating interaction with the external environment [1]. It turns out that almost $10 \%$ of genes coding proteins contain a significant change such as an altered reading frame or a premature stop codon [1]. Nevertheless, the extent to which adaptation to a specific region is reflected in the phenotype of particular plant ecotypes is still poorly understood and the precise effect of the genomic changes on 
the individual phenotype is not clear. But it is widely presumed that some of these changes contribute to increased phenotypic variability.

As with many other traits, basal hormone levels in the plant are genetically determined. Given their key regulatory function, even small, genetically determined differences can play a significant role in the control of intraspecific variability of plant development or adaptive responses. The variability of basal hormone content due to genetic divergence is not known. Further, it is unclear whether there is a relationship between increased incidence of SNPs in genes related to interactions with the environment and the particular climatic and environmental conditions prevailing in the geographical place of origin of the particular intraspecific population. In other words, it is still not clear if the differences in phenotype observed in various subpopulations of a given species (varieties, ecotypes) may be a direct result of the selective pressure on the developmental and adaptive determinants of that population.

Natural variation among ecotypes of Arabidopsis thaliana is increasingly drawing the attention of researchers [2-6]. A. thaliana is an important model plant and the knowledge of variations among ecotypes and the derived mutant plants is valuable for many researchers. Nevertheless, surprisingly little is known about differences in physiological functions and plant-environment interactions among the ecotypes and how these differences may be affected by variations in hormone levels.

Adaptation of plants to different environmental conditions is tightly connected to plant hormones as they are the principal mediators of plant developmental responses. As with other traits, differences exist among species in hormone levels and, consequently, in their response to environmental conditions [7]. It has been shown that significant differences exist in this respect not only among different species but also among individual ecotypes within the same species such as A. thaliana. Our preliminary experiments indicated that the differences exist in levels of cytokinins (CKs) and their metabolites among ecotypes even in plants grown under stable and identical environmental conditions [8]. Although hormone levels change in response to variations in the environment, the dissimilarities in inherent basal concentrations may determine potential differences in the dynamics or even in the magnitude of the response to such environmental factors. Examples of plant hormone-mediated responses to the environment without any change in hormonal level, but due to factors modulating hormonal signal such as $\mathrm{pH}$, have already been well documented for abscisic acid (ABA) [9].

In our work, we used a collection of thirty different $A$. thaliana ecotypes (Table 1 ) that represent a wide genetic variability within the species based on SNPs [1]. The group also included other ecotypes frequently used in experimental work (e.g., Ws-0, Col-0, Ler-1). We determined steady-state (basal) levels of three major plant hormones-cytokinins, ABA and auxin (indole-3-acetic acid-IAA) in this set of ecotypes grown under optimal and stable environmental conditions. We hypothesised that although a large variability exists among ecotypes in the levels of all major plant hormones even when all plants are cultivated under the same environmental conditions, significant patterns of hormone content exist within the whole group of ecotypes. Using advanced statistical methods, we analysed the extent to which hormone levels can specifically identify a particular ecotype. We also explored the possible relationships of hormone levels with the environmental conditions of the geographical site where the respective ecotype was originally collected.

Table 1. List of ecotypes used in this study including abbreviations, country and region of origin of each ecotype. Listed are also main environmental parameters for each site of origin: Mean year temperature in ${ }^{\circ} \mathrm{C}$ (Temp.), Mean total annual precipitation in $\mathrm{mm}$ (Prec.), Mean altitude (Altit.) and precise geographical location-Latitude and Longitude (Lat., Long.).

\begin{tabular}{cccccccc}
\hline Ecotype & Country & Region & Temp. & Prec. & Altit. & Lat. & Long. \\
\hline Bay-0 & Germany & Bayreuth & 8.7 & 490 & 350 & 49 & 11 \\
Bor-4 & Czech Rep. & Borky (Brno) & 8.7 & 490 & 250 & 49.4 & 16.2 \\
Br-0 & Czech Rep & Brunn (Brno) & 8.7 & 490 & 250 & 49.2 & 16.6 \\
Bur-0 & Ireland & Burren & 9.3 & 805 & 50 & 54.1 & -6.2 \\
\hline
\end{tabular}


Table 1. Cont.

\begin{tabular}{|c|c|c|c|c|c|c|c|}
\hline Ecotype & Country & Region & Temp. & Prec. & Altit. & Lat. & Long. \\
\hline $\mathrm{C} 24$ & Portugal & Coimbra & 15.7 & 1014 & 150 & 40.2 & -8.4 \\
\hline CIBC-5 & UK & Ascot, Berks & 10.4 & 594 & 50 & 51.4 & -0.6 \\
\hline Col-0 & USA & Columbia & 12.1 & 991 & 50 & 38.3 & -92.3 \\
\hline $\mathrm{Ct}-1$ & Italy & Catania & 17.3 & 547 & 50 & 37.3 & 15 \\
\hline Cvi-0 & $\begin{array}{l}\text { Cape Verde } \\
\text { Islands }\end{array}$ & Santa Cruz & 23.5 & 70 & 1150 & 15.1 & -23.6 \\
\hline $\mathrm{Ei}-2$ & Germany & Eifel & 9.6 & 933 & 450 & 50.3 & 6.3 \\
\hline Est-1 & Estonia & Tartu & 4.8 & 589 & 150 & 58.3 & 25.3 \\
\hline Fei-0 & Portugal & Santa Maria da Feira & 14.5 & 1267 & 50 & 40.9 & -8.5 \\
\hline Got-7 & Germany & Göttingen & 8.9 & 655 & 150 & 51 & 10 \\
\hline $\mathrm{Hr}-5$ & UK & Ascot, Berks & 10.4 & 594 & 50 & 51.4 & -0.6 \\
\hline Kon & Tajikistan & Khurmatov & 14.7 & 568 & 800 & 38.5 & 68.5 \\
\hline Ler-1 & Poland/Germany & $\begin{array}{c}\text { Gorzow } \\
\text { Wielkopolski/Landsberg }\end{array}$ & 7.8 & 805 & 450 & 48 & 10.9 \\
\hline Lov-5 & Sweden & $\begin{array}{c}\text { Lövvik/Sandöverken, } \\
\text { Harnosand }\end{array}$ & 3.0 & 570 & 50 & 62.8 & 18.1 \\
\hline Mt-0 & Libya & Martuba/Cyrenaika & 18.9 & 96.5 & 150 & 32.3 & 22.5 \\
\hline NFA-8 & UK & Ascot & 10.0 & 570 & 50 & 51.4 & -0.6 \\
\hline No-0 & Germany & Nossen/Halle & 9.0 & 595 & 150 & 51.1 & 13.3 \\
\hline RRS-10 & USA & North Liberty, Indiana & 9.7 & 994 & 250 & 41.6 & -86.4 \\
\hline RRS-7 & USA & North Liberty, Indiana & 9.7 & 994 & 250 & 41.6 & -86.4 \\
\hline Shahdara & Tajikistan & $\begin{array}{c}\text { Shakdara river (Pamiro } \\
\text { Alaya) }\end{array}$ & 14.7 & 568 & 3350 & 37.4 & 71.6 \\
\hline Ta-0 & Czech Rep. & Tabor & 7.8 & 526 & 450 & 49.5 & 14.5 \\
\hline Ts-1 & Spain & Tossa del Mar & 15.3 & 658 & 50 & 41.7 & 2.9 \\
\hline Tsu-1 & Japan & Tsukuba/Tsushima & 15.1 & 1535 & 50 & 34.4 & 136.3 \\
\hline Van-0 & Canada & Vancouver & 9.9 & 1167 & 50 & 49.3 & -123 \\
\hline Wei-0 & Switzerland & Weiningen & 8.5 & 1101 & 550 & 47.3 & 8.3 \\
\hline Ws-0 & Belarus & Vasil'yevka & 6.5 & 588 & 150 & 52.3 & 30 \\
\hline Ws-2 & Belarus & Vasil'yevka & 6.5 & 588 & 150 & 52.3 & 30 \\
\hline
\end{tabular}

\section{Results and Discussion}

\subsection{Inherent Variation in Hormone Content}

Similarly to other compounds, also hormonal content in the tissue can be influenced by the relative contribution of various cell types. Therefore, it is also influenced by the morphology and developmental stage of the plant body and the inherent differences in the rates of growth and development should be considered when comparing the set of ecotypes. Our preliminary experiments, however, showed that in the early stage of growth the differences in size and morphology among ecotypes are small. Only a few ecotypes were significantly different in the number of leaves (Br-0, Cvi-0, RRS-10, Tsu-1) than the rest of the tested group (Figure S1A). Similarly, only a few ecotypes significantly differed in root length (Figure S1B). Additionally, we found no statistically significant correlation between hormonal content in selected ecotypes and either ecotype morphology or inherent developmental rate of the ecotype.

Given that the plants were grown under identical and stable cultivation conditions resulting in uniform growth rate and development/morphology of ecotype majority, the variability in hormone contents that we observed among the ecotypes was surprisingly high. The content of CKs varied between 150 to $500 \mathrm{pmol} \mathrm{g}^{-1}$ in both shoot and root (Figure 1). Interestingly, the Ws-0 ecotype that has frequently been used in genomic analyses and other experimental work had the smallest content of CKs in both root and shoot of all the examined ecotypes and appeared to be an extreme case in this respect. Variation in CK content was comparable in shoot and root and ecotypes with high CK content usually showed similar levels in both organs (e.g., Hr-5, Ct-1). It was previously shown that high CK content, particularly in the shoot, may be beneficial for growth and drought stress tolerance [10]. In this context, 
by far the most frequently used ecotype-Col-0 had the highest proportion of basal CK concentration in the shoot of all the ecotypes examined (Figure S3A). Seventy per cent of examined ecotypes had a higher $\mathrm{CK}$ content in roots, which fits well with previous observations suggesting a dominant role for roots in CK biosynthesis [11-13]. Generally, we found only a rather small variation of the ratio of CKs contents in the root and shoot in our set of ecotypes (Figure S3A). A possible explanation for this could be similar transport rates of root-derived CKs to the shoot in the majority of ecotypes. Interestingly, Col-0 revealed the lowest root-to-shoot ratio, being a consequence of both low root and high shoot CK content measured in Col-0 seedlings.
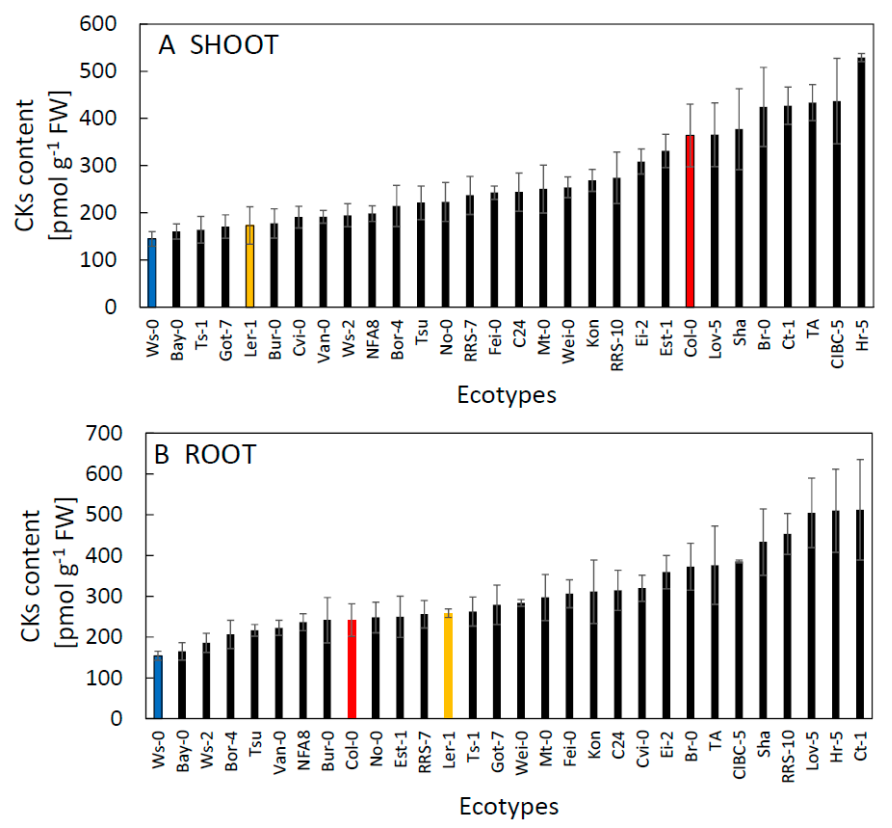

Figure 1. The content of CKs in shoot (A) and root (B) of thirty A. thaliana ecotypes grown $21 \mathrm{~d}$ in controlled conditions. Commonly used ecotypes are marked in colour (Red $=$ Col- 0 , Blue $=$ Ws -0 , Orange $=$ Ler-1). See Table 1 for the full list of ecotypes. Means \pm SD.

Compared to CKs, the content of IAA differed considerably between the shoot and root (Figure 2, Figure S3B). In shoots, the IAA content varied between 80 and $250 \mathrm{pmol} \mathrm{g}^{-1}$ whereas in roots the amount of IAA was much greater, typically between 220 to $1000 \mathrm{pmol} \mathrm{g}^{-1}$, also with greater variation (approx. 4.5 fold) than in the shoot. Interactions of IAA and CKs are currently a target of intensive research [14]. It has long been known that the ratio of auxin to cytokinin determines the differentiation of plant tissues $[15,16]$. We tested the possible importance of the relationship between IAA and CK contents among the ecotypes. Although the ratio varied more than seven-fold (Figure S3) there was no significant correlation either in shoots $(\mathrm{r}=-0.288 ; p=0.1228)$ or in roots $(\mathrm{r}=-0.2374 ; p=0.2065)$. In general, some ecotypes showed systematically higher levels (in the range of upper 50\% among ecotypes examined) of all analysed hormones in both shoots and roots (Mt-0, Wei-0, Ta-0, RRS-10, Sha), whereas others showed systematically lower levels (lower 50\% among ecotypes examined-Bor-4, Col-0, Ts-1, Ler-1). The highest levels of IAA typically associated with the highest IAA/CK ratios (Figure 2, Figure S3).

The content of ABA in both shoot and root was low, typically ranging between 5 and $30 \mathrm{pmol} \mathrm{g}^{-1}$ with an approximately ten-fold variation among ecotypes (Figure 3). We observed greater variation in the shoot. Generally, low ABA contents are probably related to high water availability and high humidity inside cultivation dishes [17]. In many ecotypes, the slightly higher ABA levels in the root were also accompanied by higher ABA content in the shoot. About two-thirds of the ecotypes showed higher ABA content in roots compared to shoots (Figure S2C), which fits with the view that roots are the dominant source of ABA as well. Moreover, in five of the ecotypes the ABA content in the root was more than double that in the shoot (Got-7, Tsu, NFA8, Ta-0, Sha; Figure S1C). Elevated ABA levels only in the root (but not in the shoot) were 
previously shown to be beneficial for plant productivity since both the hydraulic conductance of roots and stomatal conductance are high, and that favours plant gas exchange [18]. Elevated ABA in the root may also support plant drought tolerance, as suggested by Ghanem et al. [19].
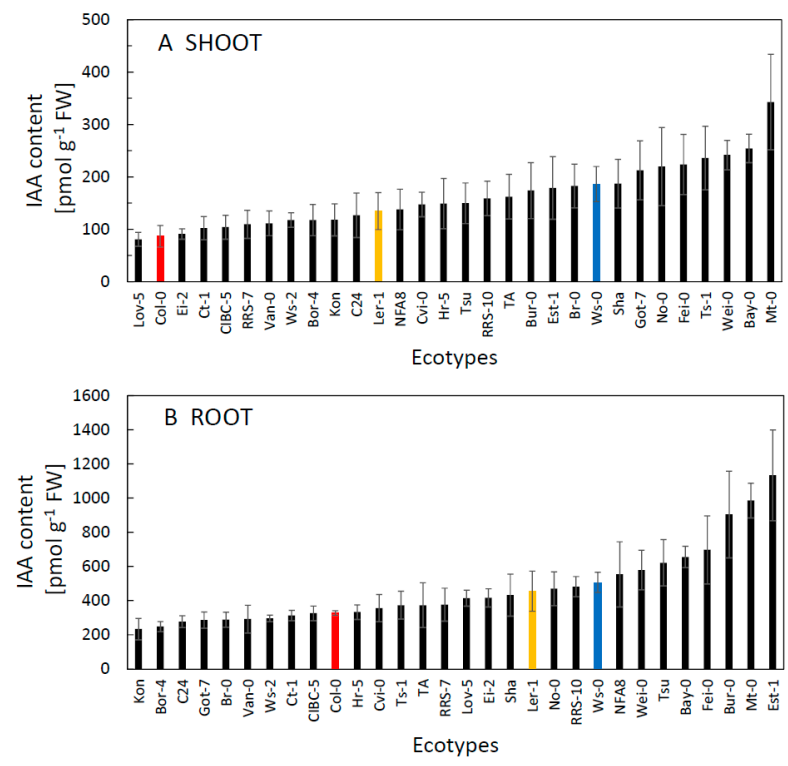

Figure 2. The content of IAA in the shoot (A) and $\operatorname{root}(\mathbf{B})$ of thirty $A$. thaliana ecotypes grown $21 \mathrm{~d}$ in controlled conditions. Commonly used ecotypes are marked in colour (Red $=$ Col-0, Blue $=$ Ws- 0 , Orange $=$ Ler-1). See Table 1 for the full list of ecotypes. Means \pm SD.

In ecotypes with high levels of IAA, we frequently observed lower levels of ABA (Figures 2 and 3) most likely as a result of the mutually negative interactions between metabolic pathways of the two hormones [20]; however, this trend was not significant. Therefore, we also examined the variation in the ratio of IAA and ABA among the ecotypes (Figure 4). The interplay between IAA and ABA can affect the development of the root system, thereby influencing plant (drought) stress tolerance [19]. The ratio reflected considerably higher content of IAA, and it varied about fivefold. In some ecotypes (Ta-0, Cvi-0), however, the ratio was considerably shifted in favour of ABA content (Figure 4).
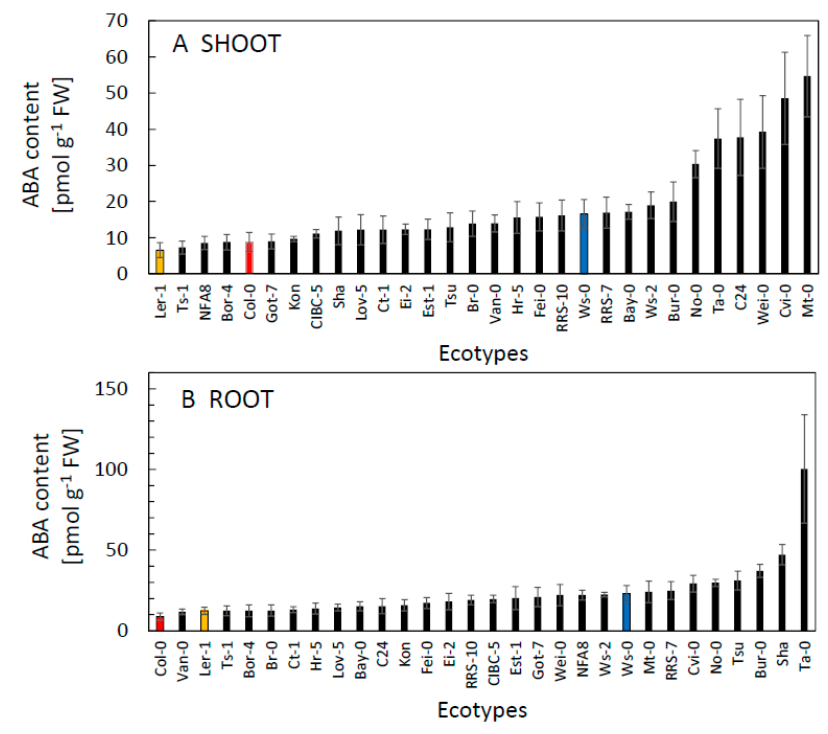

Figure 3. The content of ABA in the shoot (A) and root (B) of thirty A. thaliana ecotypes grown 21d in controlled conditions. Commonly used ecotypes are marked in colour (Red $=$ Col-0, Blue $=$ Ws- 0 , Orange $=$ Ler-1). See Table 1 for the full list of ecotypes. Means \pm SD. 

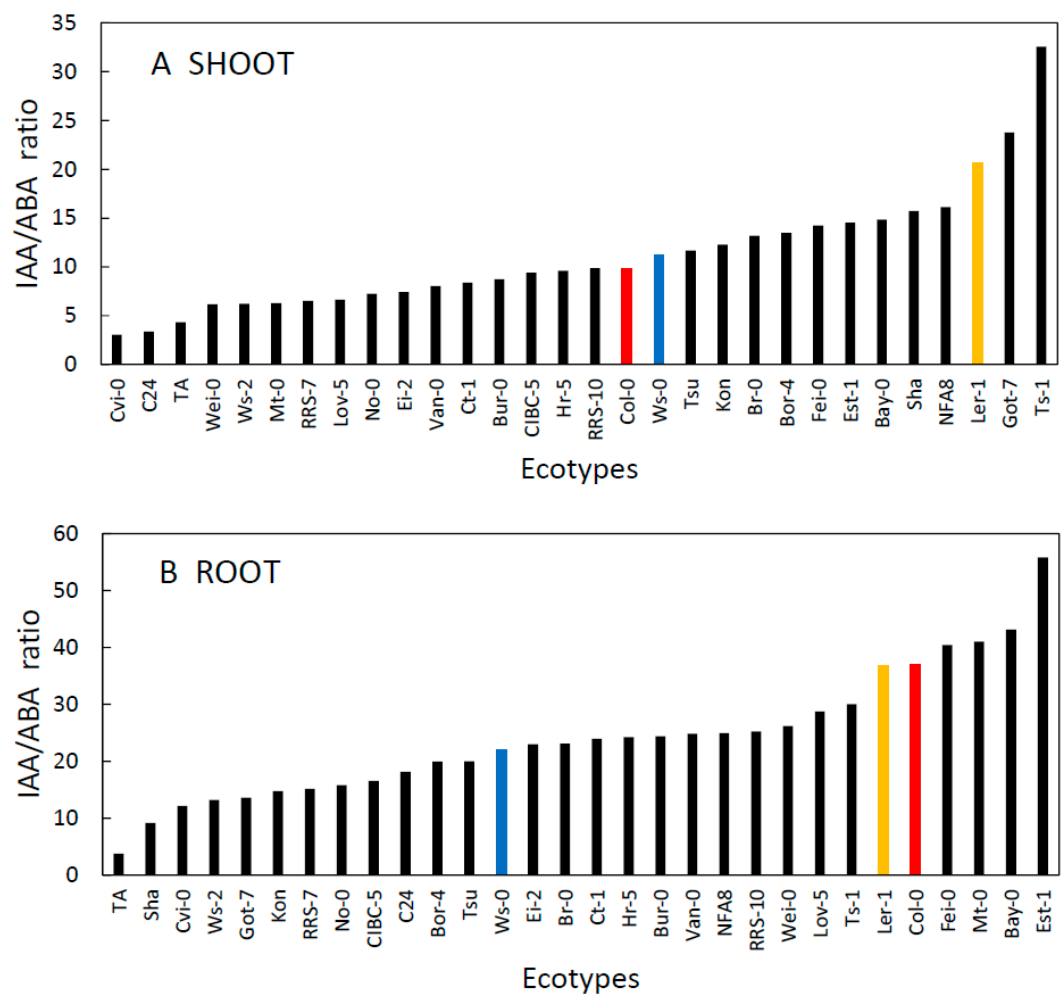

Figure 4. The ratio of IAA to ABA content in the shoot $(\mathbf{A})$ and root $(\mathrm{B})$ ranked from the lowest to the highest among thirty $A$. thaliana ecotypes grown $21 \mathrm{~d}$ in controlled conditions. Commonly used ecotypes are marked in colour $($ Red $=$ Col- 0 , Blue $=$ Ws- 0 , Orange $=$ Ler- 1$)$. See Table 1 for the full list of ecotypes.

\subsection{Hormone Levels as an Ecotype-Specific Trait}

Using several statistical approaches we explored the significance of basal hormonal levels and their ratios in roots and shoots as a specific identifier of the ecotype. The Random Forest classifier with the significance measure of Mean Decrease in Impurity (MDI) [21] showed that at the level of total hormone content, $\mathrm{CKs}$ and less so ABA (specifically for the Ta-0 ecotype) can be significant identifiers for a small number of ecotypes (see Appendix A, Tables A1 and A2). In the next step of our analysis, we used the complete data set including all metabolites in the CKs group. This approach produced much more precise and significant results.

Classification analysis revealed that glycosylation contributes significantly to the setting of ecotype-specific CK levels in both roots and shoots during early seedling development. CK glucosides have the highest accuracy even when a similar number of trait variables are considered for classification (see Table 2). The classification of ecotypes was significantly improved for both shoots and roots when glucosides were included in the analysis and improved classification can also be inferred from the fact that CK glucosides seem to play the most important role in the final Random Forest classifier (Table 2). The total classification accuracy of the classifier (using repeated hold-out cross-validation) was $72.1 \%$ in roots and $72.9 \%$ in shoots (Table 2). Furthermore, the importance of individual metabolites for classification can be seen from the MDI index (Table 3). The finding that iPR is an important classifier in both shoots and roots (Table 3) provides support to the hypothesis that this compound is the principal form in which root-synthesized CKs are delivered to the shoot [22-27]. 
Table 2. Overall classification accuracy of the ecotypes using Random Forest classifier with different traits using hold-out cross validation. Number of compounds used for analysis is stated in brackets.

\begin{tabular}{ccc}
\hline Trait Variables Used in the & \multicolumn{2}{c}{ Accuracy (\%) } \\
\cline { 2 - 3 } Random Forest as Predictors & Roots & Shoots \\
\hline ABA, total CKs, IAA (3) & $28.5 \%$ & $32.1 \%$ \\
CK non-glucosides (10) & $38.4 \%$ & $41.9 \%$ \\
CK glucosides (12) & $68.8 \%$ & $66.5 \%$ \\
All CKs (22) & $72.1 \%$ & $72.9 \%$ \\
\hline
\end{tabular}

Table 3. The importance of various cytokinin derivatives in root and shoot as trait variables for classification based on the Mean Decrease in Impurity value (MDI). The most important compounds are on the top of the list. Abbreviations: trans-zeatin ( $t \mathrm{Z})$, trans-zeatin riboside $(t \mathrm{ZR})$, trans-zeatin-O-glucoside ( $t Z O G)$, trans-zeatin-O-glucoside riboside ( $t Z R O G)$, trans-zeatin N7-glucoside $(t Z 7 G)$, trans-zeatin N9-glucoside ( $t Z 9 G)$, trans-zeatin riboside-5' -monophosphate ( $t Z R M P)$, cis-zeatin $(c Z)$, cis-zeatin riboside (cZR), cis-zeatin-O-glucoside (cZOG), cis-zeatin-O-glucoside riboside (cZROG), cis-zeatin N9-glucoside (cZ9G), cis-zeatin riboside-5'-monophosphate (cZRMP), dihydrozeatin (DHZ), dihydrozeatin riboside (DHZR), dihydrozeatin-O-glucoside (DHZOG), dihydrozeatin N7-glucoside (DHZ7G), dihydrozeatin N9-glucoside (DHZ9G), isopentenyladenosine (iPR), isopentenyladenine $N 7$-glucoside (iP7G), isopentenyladenine $N 9$-glucoside (iP9G), isopentenyladenine riboside-5' -monophosphate (iPRMP).

\begin{tabular}{cccc}
\hline ROOT & MDI & SHOOT & MDI \\
\hline cZROG & 3.98783 & cZROG & 5.28804 \\
iP7G & 3.93474 & iPRMP & 4.60337 \\
iPR & 3.71329 & iP7G & 4.45139 \\
tZROG & 3.5989 & iPR & 4.37966 \\
DHZ7G & 3.58955 & tZROG & 3.99245 \\
cZR & 3.39747 & DHZOG & 3.87761 \\
tZRMP & 3.35821 & cZ9G & 3.80151 \\
cZOG & 3.305 & DHZ9G & 3.62936 \\
DHZOG & 3.2893 & iP9G & 3.48557 \\
tZOG & 3.00218 & DHZ7G & 3.33904 \\
DHZ & 3.0011 & tZRMP & 3.3029 \\
tZR & 2.9322 & tZR & 3.20053 \\
cZ9G & 2.93091 & iP & 3.16696 \\
tZ7G & 2.8261 & tZ7G & 3.01294 \\
tZ9G & 2.72127 & tZ9G & 2.97171 \\
cZRMP & 2,45831 & DHZ & 2.95543 \\
DHZ9G & 2.399 & cZR & 2.86284 \\
iPRMP & 2.39615 & tZOG & 2.80756 \\
cZ & 2.22799 & DHZR & 2.62581 \\
DHZR & 2.12067 & tZ & 2.46915 \\
tZ & 2.08467 & cZRMP & 2.44495 \\
iP9G & 2.039 & cZOG & 2.27142 \\
\hline
\end{tabular}

It is well known that even minor local and transient changes in CK levels can have profound physiological effects [36-39]. Thus it is highly likely that the specificity of CK action depends to a significant extent on the determination setting of CK levels with contributions from the entire network of CK conversions. Therefore, the diverse mechanisms that affect this determination must be considered to get a full picture of the $\mathrm{CK}$ role in any physiological response. Such mechanisms include not only the conversions of CK from one form to another but also other mechanisms underlying the availability of individual CK substrate forms and their cognate enzymes. Furthermore, the CK homeostatic mechanisms are very dynamic and include processes such as cell-to-cell transport and 
sub-cellular sequestration or compartmentation of CK metabolites as well as genetic variability of TCS components which in turn further modulate the primary CK response.

Our study lays the groundwork for a more detailed exploration of the contributors to the ecotype-specific levels of CKs observed in our study. Our data indicate that the activities of enzymes that are responsible for CK metabolic conversions, particularly those responsible for glycosylation/deglycosylation, vary in an ecotype-specific manner, and this variation gives rise to the observed importance of glucosides as the strongest classifier. Further, it is highly likely that ecotype-specific variations exist in the sensitivity to CKs, and a thorough explication of this variability could form part of a future analytical study.

\subsection{Hormone Levels and the Site of Origin of Ecotype}

Besides the inherent hormonal settings among ecotypes, we also explored the relationship between basal hormonal levels and the geographical location and environmental conditions prevalent in the sites of origin of the respective ecotypes.

Plant response to stress is frequently associated with an increase in the content of $\mathrm{ABA}$ and also the ratio between $\mathrm{ABA}$ and $\mathrm{CKs}$ that combine to regulate stomatal aperture size [40,41]. This shift in hormonal content contributes to the balance between plant growth rate and availability of resources (e.g., water, nutrients), particularly under stress-inducing conditions [42]. We indeed found a large variation in the CK/ABA ratio among ecotypes in both shoots and roots (Figure 5). Detailed analysis of data discovered more significant relationships at the organ level. There was a positive correlation of mean ABA content in the shoot with the mean temperature at the site of origin $(r=0.404, p=0.02688$; Figure $6 \mathrm{~A}$ ). The correlation between the $\mathrm{ABA} / \mathrm{CK}$ ratio in the shoot and the site temperature was also significant $(r=0.4317, p=0.0172$, Figure $6 \mathrm{~B})$. Moreover, there was also a significant positive correlation between the ABA shoot content and altitude above sea-level of the site of origin $(r=0.4, p=0.031)$; however, this relationship was most likely mediated by the strong correlation between the altitude and the mean site temperature. Effects of the temperature at the site of origin on basal hormonal balance can be further highlighted by classifying the sites based on mean temperature (Figure 7). We labelled the sites as WARM $\left(\mathrm{T}>15.5^{\circ} \mathrm{C}\right)$ and $\operatorname{COLD}\left(\mathrm{T}<15.5^{\circ} \mathrm{C}\right)$. Results of a t-test and a Welch test revealed that places with a mean temperature higher than $15.5^{\circ} \mathrm{C}$ tend to have higher mean $\mathrm{ABA}$ values for the shoots ( $p=0.08$ for both tests). No other correlations of basal hormone levels with geographical and environmental factors showed any significant relationships (Table S1).
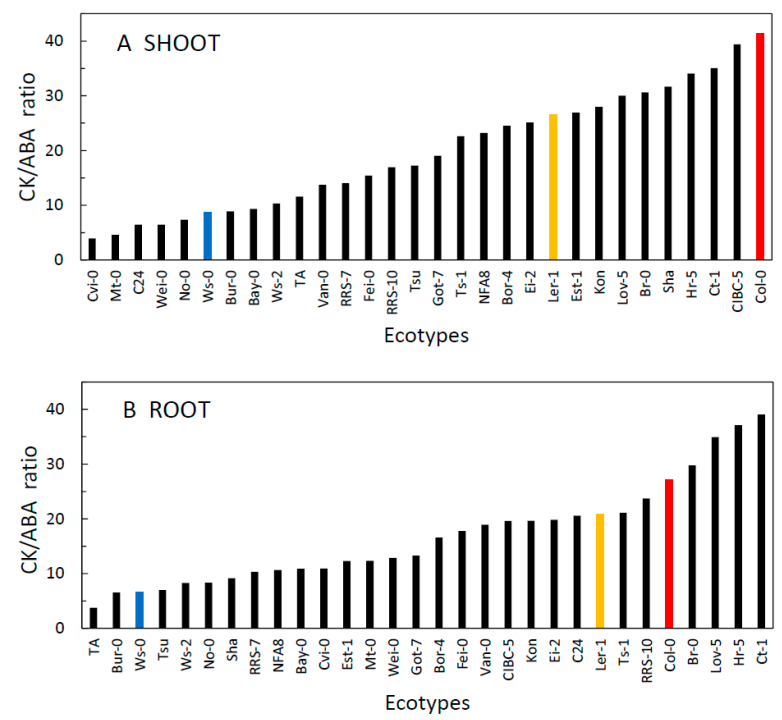

Figure 5. The ratio of total CKs to ABA in the shoot (A) and root (B) ranked from the lowest to the highest among thirty $A$. thaliana ecotypes grown $21 \mathrm{~d}$ in controlled conditions. Commonly used ecotypes are marked in colour $($ Red $=$ Col-0, Blue $=$ Ws-0, Orange $=$ Ler-1). See Table 1 for full list of ecotypes. 


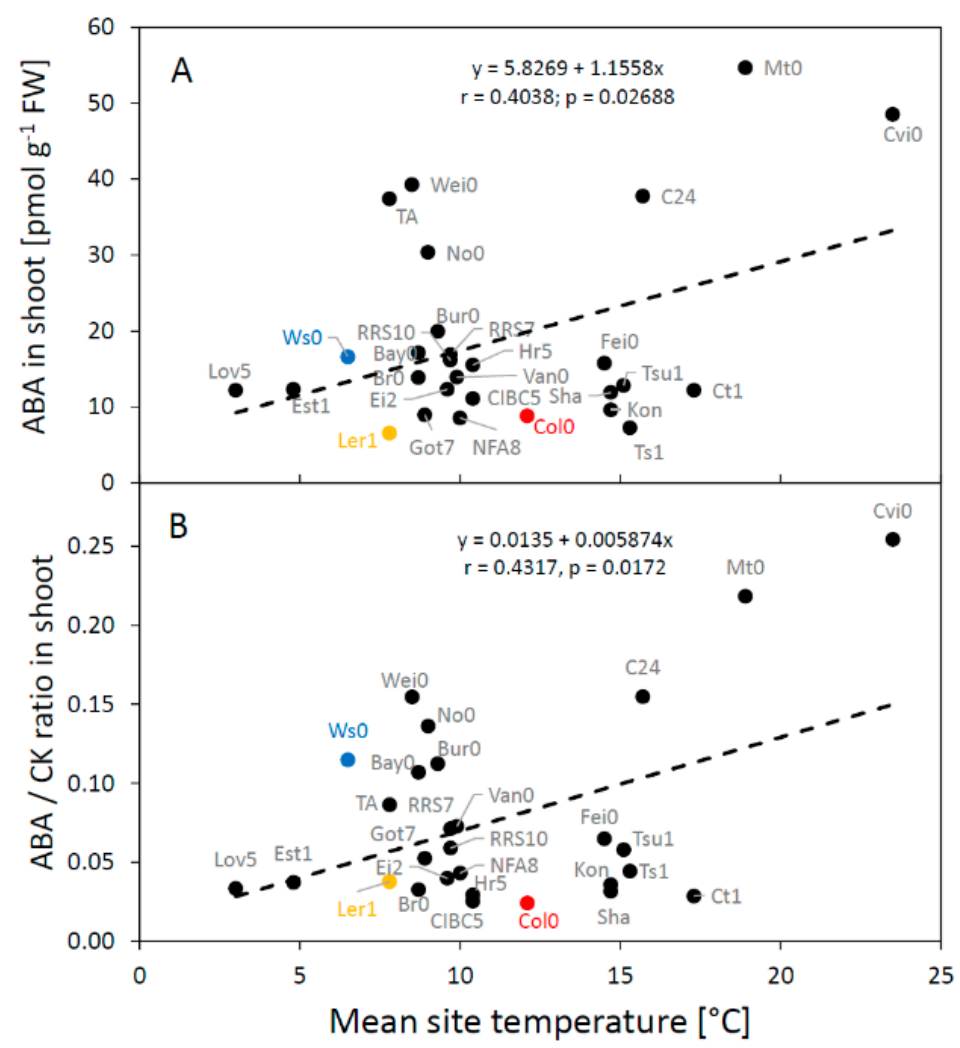

Figure 6. Relationships between the mean temperature at the site of ecotype origin and basal level of abscisic acid (ABA) content in the shoot $(\mathrm{A})$ and the ratio between ABA and total cytokinin content in the shoot (ABA/CK ratio) in the shoot (B) in A. thaliana ecotypes. Commonly use ecotypes are marked in colour $($ Red $=$ Col-0, Blue $=$ Ws- 0 , Orange $=$ Ler-1). See Table 1 for the full list of ecotypes.

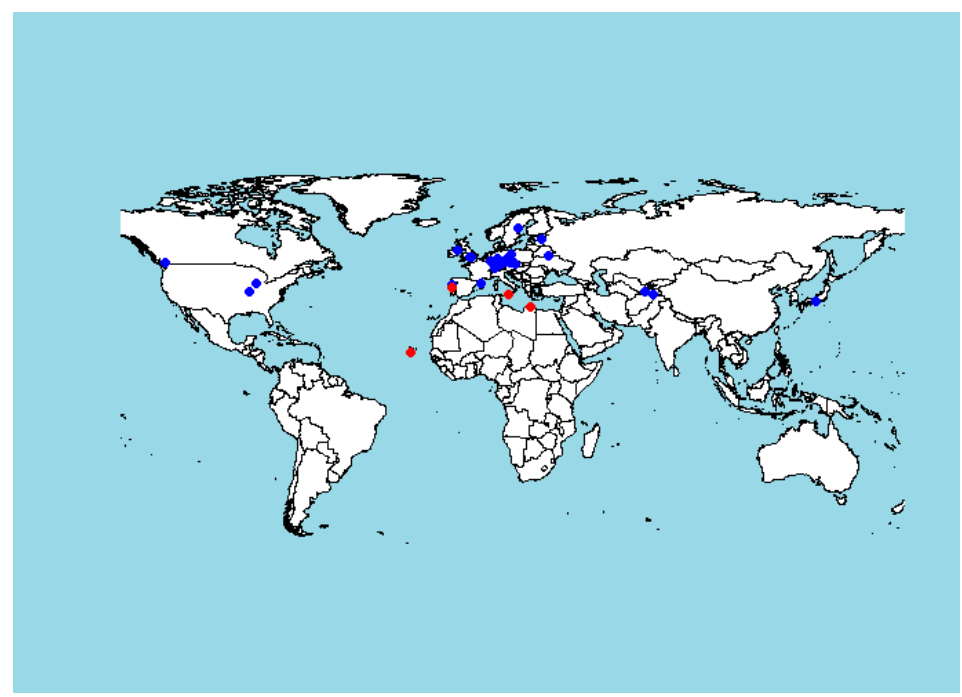

Figure 7. World map showing locations of sites of origin for the examined A. thaliana ecotypes. Colors indicate classification of sites based on the mean temperature: Red: WARM $\left(\mathrm{T}>15.5^{\circ} \mathrm{C}\right)$, Blue: COLD $\left(\mathrm{T}<15.5^{\circ} \mathrm{C}\right)$. The map was generated in R package (v. 3.3.0) using the package "Draw Geographical Maps" (2018). (https://cran.r-project.org/web/packages/maps/). 


\section{Materials and Methods}

\subsection{Plant Material}

A collection of 30 selected ecotypes of Arabidopsis thaliana based on observations by Clark et al. (2007) was used in this study (Table 1) [1]. Tamm-2 from the original Clark collection was not included due to unsuccessful seed propagation. Seeds were obtained from NASC (Nottingham Arabidopsis Stock Centre, Nottingham, UK) and propagated in stable greenhouse conditions with supplemented light.

\subsection{Plant Cultivation and Sampling}

Before sowing, all seeds were sterilized for $5 \mathrm{~min}$ in a solution of $75 \%$ EtOH with $0.1 \%$ Triton-X-100 and dried thoroughly. Seeds of each ecotype were sown separately in square Petri plates $(12 \times 12 \mathrm{~cm})$, 15 seeds per plate and 20 plates per ecotype.

The cultivation medium was half-strength Murashige and Skoog (MS) without sucrose that contained: MS macro and microelements (Duchefa, Haarlem, The Netherlands) with MES buffer (500 $\mathrm{mg} \mathrm{L}^{-1}$ ) in redistilled water; $\mathrm{pH}$ was corrected by $1 \mathrm{M} \mathrm{KOH} \mathrm{(5.7-5.8).} \mathrm{The} \mathrm{medium} \mathrm{was} \mathrm{solidified}$ using $1.2 \%$ agar.

A sterile fabric with ultrafine mesh (Uhelon, Silk \& Progress, Brněnec, CZ) was placed on the plated, solidified medium a few hours before sowing to facilitate harvesting. After sowing, the plates were sealed with vapour permeable tape and stored at $4{ }^{\circ} \mathrm{C}$ in the dark for three days for stratification. On the fourth day, plates were placed vertically in a cultivation box with stable environmental conditions: photosynthetic photon flux density $120 \mu \mathrm{mol} \mathrm{m} \mathrm{m}^{-2} \mathrm{~s}^{-1}$ supplied with fluorescent lamps (Osram Fluora TLD 36W, Osram AG, Munich, Germany) with photoperiod $16 \mathrm{~h}$, temperature $21 / 19^{\circ} \mathrm{C}$ day/night and air humidity 40 and 50\% during day and night respectively.

Plants were harvested on the 21st day after sowing (DAS). The timing of harvest was based on our preliminary experiments showing only minor differences among ecotypes in morphology 14 to 21 DAS (Figure S1) and, simultaneously, providing a sufficient amount of biomass for analyses. Plants typically had 6 to 8 leaves and root systems with only a small number of short lateral roots. Roots and shoots were weighed separately and immediately flash-frozen in liquid nitrogen and stored at $-80{ }^{\circ} \mathrm{C}$ until hormone quantification. Means of 3-4 replicate samples per organ and ecotype (biological replicates) were analysed. Each sample contained several shoots (3-8) or roots (20-30).

\subsection{Quantification of Hormones}

Small amounts of tissue $(20 \mathrm{mg})$ were homogenized and extracted in $1 \mathrm{~mL}$ of modified Bieleski buffer $\left(60 \% \mathrm{MeOH}, 10 \% \mathrm{HCOOH}\right.$ and $\left.30 \% \mathrm{H}_{2} \mathrm{O}\right)$ together with a cocktail of stable isotope-labeled internal standards ( 0.25 pmol of CK bases, ribosides, $N$-glucosides, 0.5 pmol of CK O-glucosides and nucleotides, 5 pmol of ${ }^{2} \mathrm{H}_{5}$-IAA and ${ }^{2} \mathrm{H}_{5}$-ABA per sample). The extracts were purified using two solid-phase extraction columns, an octadecylsilica-based column (C18, $500 \mathrm{mg}$ of sorbent, Applied Separations) and after that an Oasis MCX column (30 mg/1 mL, Waters, Milford, MA, USA) [43]. Analytes were eluted by a three-step elution using $60 \%(v / v) \mathrm{MeOH}, 0.35 \mathrm{M} \mathrm{NH}_{4} \mathrm{OH}$ aqueous solution and $0.35 \mathrm{M} \mathrm{NH}_{4} \mathrm{OH}$ in $60 \%(v / v) \mathrm{MeOH}$. Samples were subsequently analysed using an ultra-high performance liquid chromatography (UHPLC) system coupled to a tandem quadrupole mass spectrometer (MS/MS) equipped with an electrospray interface (ESI) [44,45]. Quantification was performed by Masslynx software (Waters) using a standard isotope dilution method with stable isotope-labelled internal standards as a reference.

The following hormones and their derivatives were quantified: IAA, ABA, CKs. IAA was chosen as it is the predominant natural form of auxin. CKs were presented as a sum of all major and minor components detected in each of their metabolic groups (bases, ribosides, nucleotides, $O$-glucosides, $N$-glucosides. Namely: trans-zeatin $(t Z)$, trans-zeatin riboside ( $t Z R)$, trans-zeatin-O-glucoside $(t Z O G)$, trans-zeatin-o-glucoside riboside ( $t Z R O G)$, trans-zeatin N7-glucoside ( $t Z 7 G)$, trans-zeatin N9-glucoside $(t Z 9 G)$, trans-zeatin riboside-5'-monophosphate (tZRMP), cis-zeatin (cZ), cis-zeatin riboside (cZR), 
cis-zeatin-O-glucoside (cZOG), cis-zeatin-O-glucoside riboside (cZROG), cis-zeatin N9-glucoside (cZ9G), cis-zeatin riboside-5'-monophosphate (cZRMP), dihydrozeatin (DHZ), dihydrozeatin riboside (DHZR), dihydrozeatin-O-glucoside (DHZOG), dihydrozeatin-O-glucoside riboside (DHZROG), dihydrozeatin N7-glucoside (DHZ7G), dihydrozeatin N9-glucoside (DHZ9G), dihydrozeatin riboside-5'-monophosphate (DHZRMP), isopentenyladenine (iP), isopentenyladenosine (iPR), isopentenyladenine N7-glucoside (iP7G), isopentenyladenine N9-glucoside (iP9G), isopentenyladenine riboside-5'-monophosphate (iPRMP).

\subsection{Statistical Analysis and Ecotype Classification Using Random Forest}

Possible relationships among various hormones and between hormone levels and environmental factors were tested by correlation analysis using the Pearson correlation coefficient. Environmental factors involved in the analysis were: Average precipitation (spring/autumn), average temperature (spring/autumn), mean day-length, geographical location on the globe (latitude and longitude) and elevation above sea level. The data used were obtained for sites in the closest proximity to the site of origin of respective ecotype where the data were freely available. Significance of differences among sites with different temperatures was also tested by $t$-test. Despite the fact that the samples are extremely small, the effect sizes are large and thus, we examined differences using both $t$-test and Welch test (unequal variance $t$-test) [46,47]. The classification analysis was based on the Random Forest classifier method. This method is particularly useful when there are more than two categories in a response variable-e.g., ecotypes [21,48]. A more detailed description of the methodology of classification analysis is provided in the Supplementary Materials. All statistical tests were performed using the $\mathrm{R}$ software [49].

\section{Conclusions}

We found significant variations in the basal hormone levels among a wide range of $A$. thaliana ecotypes. Considering the previously demonstrated role of hormonal regulation in stress response [7], the large variability in basal hormone levels, as well as some significant relationships with temperature as a key environmental factor, might imply inherent differences among ecotypes in drought stress tolerance [50]. We also clearly showed that CK levels-uniquely among the hormones tested can be used as an ecotype classifier. These ecotype-specific levels of CKs might further affect the two-component system (TCS) to regulate the primary response of $A$. thaliana to internal as well as to environmental stimuli. Nevertheless, the actual CK pool does not reflect the activity of TCS, which carries SNPs among the contrasting ecotypes to fine-tune CK signalling, suggesting avenues for further research of the natural genetic variability of TCS components. Natural polymorphism in TCS components might substantially affect the final CK response as shown for instance in mutagenic studies on CK sensor histidine kinases, which identified mutations leading to either impaired kinase activity or conversely to CK-independent activation [51-54].

The role of CK-glucosides in the regulation of plant growth and development is still unclear. Our data, however, suggest that the content of CK-glucosides might be a trait specific to individual ecotypes. The pool of CK-glucosides that can potentially contribute to levels of active CK appears to be larger than previously expected. The generally accepted hypothesis that $N$-glucosylation irreversibly inactivates CKs was recently put into question [55]. Thus, release of active CKs from CK glucosides including the $\mathrm{N}$-glucosides, may substantially affect the whole metabolic pattern. Our study highlights the necessity of investigations on the functional importance and underlying molecular mechanisms of genetic variability of CK metabolism, particularly CK glucosylation as well as conversions of CK glucosides to free CKs.

Supplementary Materials: The following are available online at http://www.mdpi.com/2223-7747/9/1/116/s1: Table S1. Calculated non-significant correlations between hormonal levels and environmental factors at the site of origin of the ecotype; Figure S1. The number of true leaves (A) and length of main root (B) of selected ecotypes 
measured in a smaller preliminary experiment. Figure S2. The ratio of contents of CKs, IAA and ABA in root and shoot; Figure S3. The ratio of IAA and total CKs in the shoot and the root.

Author Contributions: Conceptualization and design, Z.S., N.S.K. and V.G.; plant cultivation and sample preparation, Z.S.; sample analysis and evaluation, O.N.; basic data analysis, Z.S., V.G.; statistical data analysis, I.S.; writing-Original draft preparation, V.G., Z.S.; writing-Review and editing, N.S.K., I.S., J.S., J.H. and V.G. All authors have read and agreed to the published version of the manuscript.

Funding: This research was funded by the Ministry of Education, Youth and Sports of the Czech Republic (National Program for Sustainability I, grant no. LO1204). This work was supported from European Regional Development Fund-Project "SINGING PLANT" (No. CZ.02.1.01/0.0/0.0/16_026/0008446) which received a financial contribution from the Ministry of Education, Youths and Sports of the CR in the form of special support through the National Programme for Sustainability II funds. This work was also supported by the project of specific research provided by the Masaryk University.

Acknowledgments: The authors give sincere thanks to Michaela Mrvková for her help with phytohormone analyses.

Conflicts of Interest: The authors declare no conflict of interest.

\section{Appendix A}

\section{Appendix A.1 Classification Analysis with the RANDOM FOREST Method}

The Random Forest classifier is a popular classification method when there are more than two categories in the response variable (ecotypes). Random Forest is a combination of many different decision trees. A decision tree is a graphical representation of all the possible solutions to a decision based on certain conditions. It is called a decision tree because it starts with a single box (called root), which then branches off into several solutions, just like a tree.

Each decision tree concludes to an outcome and the class which appears most often is the final estimated class of the Random Forest. More information about this classification method can be found in $[21,48]$.

The individual ecotypes were used as the response variable. It consisted of 29 categories:

"NFA8" "Ws-0" "Bay-0" "No-0" “Tsu" "Ler-0" "Bor-4" “Van-0" "RRS_7" “Ws-2" "Wei-0" “Got-7" “RRS-10" “Kon" “Cvi-0” “Bur-0" “Fei-0” “Ei-2" “Col-0” “Lov-5” “Est-1" “Ts-1” “Sha” “Ta-0” “Mt-0” "Br-0" "Ct-1" "C24" "Hr-5".

The explanatory trait variables (contents of hormones) used in the first analysis performed were contents of the CK, ABA and IAA and the analysis was run separately for root and shoot analytical data. The dataset consisted of 235 observations. These were divided into root (R: 116 observations) and shoot (S: 118 observations). A major advantage of the Random Forest classifier is that it provides accuracy and information about the importance of the trait variable. The most commonly used measure for variable importance is the Mean Decrease in Impurity (MDI). MDI calculates the importance of each trait variable as the sum over the number of splits (across all trees) that include the trait variable, proportionally to the number of samples it splits [48]. In addition, Random Forest can work under complex situations, e.g., when strong correlations exist among trait variables. The procedure (algorithm-like description) for estimating accuracy measures was as follows:

- Split dataset into training and test set (contains output from each category of the ecotypes variable)

- Use the training set to "train" (learning procedure) the Random forest

- Identify importance with the use of Mean Decrease in Impurity (MDI)

- Use the test set to examine the prediction accuracy of the model and fill the confusion matrix.

- Save the results

- $\quad$ Repeat this procedure 100 times with reshuffled training and test sets.

Using True Positive (TP), True Negative (TN), False Positive (FP) and False Negative (FN) predictions we can obtain useful accuracy measures as the Sensitivity and the Positive Predictive Value (PPV). 
Sensitivity is the ability of a test to correctly classify the positive values. In this case, we will evaluate as positive values the Bay- 0 , then the Ws- 0 and then the Ws- 2 and Ta- 0 :

$$
\text { Sensitivity }=\frac{\mathrm{TP}}{\mathrm{TP}+\mathrm{FN}}
$$

PPV is the percentage of ecotypes with a positive test which are correct. This means, for example, when PPV is $95 \%$ then there is $95 \%$ probability that the result is indeed positive:

$$
\mathrm{PPV}=\frac{\mathrm{TP}}{\mathrm{TP}+\mathrm{FN}}
$$

The results of the hold-out cross-validation show that the ecotypes that can be identified with good percentages by these variables were Bay-0, Ta-0, Ws-0, Ws- 2 .

Appendix A.2 Random Forest Classification for Basic Hormonal Data

The results of the first analysis:

Table A1. Random forest classification for root data. Mean decrease in impurity as a measure of significance for roots.

\begin{tabular}{ccc}
\hline & Mean MDI & Variance MDI \\
\hline IAA & 27.1 & 0.560 \\
CKs & $\mathbf{2 7 . 6}$ & 0.453 \\
ABA & 27.4 & 0.556 \\
\hline
\end{tabular}

Conclusively, Bay-0 had a $97 \%$ sensitivity and $73 \%$ PPV, Ws- -0 had $71 \%$ sensitivity and $81.6 \%$ PPV, Ws- 2 has $82 \%$ sensitivity and PPV of about $78.8 \%$ and Ta- 0 has $73 \%$ Sensitivity and $98.6 \%$ Sensitivity (This is because of the unique ABA values). However, it seems that the classifier was confused during process of identification the rest ecotypes, which resulted in a low overall accuracy $(<30 \%)$.

Table A2. Random forest classification for shoot data. Mean decrease in impurity as a measure of significance for shoots.

\begin{tabular}{ccc}
\hline & Mean MDI & Variance MDI \\
\hline IAA & 25.55 & 0.225 \\
CKs & $\mathbf{2 7 . 1 8}$ & 0.207 \\
ABA & 26.44 & 0.197 \\
\hline
\end{tabular}

Here, Ta- 0 has $97 \%$ Sensitivity and $92.3 \%$ PPV and Mt- 0 (almost unique IAA values) $69 \%$ Sensitivity and $87.34 \%$ PPV. Other results with high Sensitivity are for Hr-5 (83\%) but with low PPV (49\%) and for Ws-0 (86\%) but again with low PPV (55\%). Similarly to root data, also here the classifier was confused during the process of identification the rest ecotypes, which resulted in a low overall accuracy $(32.1 \%)$.

\section{Appendix A.3 Random Forest Classification for Using All Analyzed Metabolites and Glucosides}

In contrast, when we used the complete data set of all metabolites and glucosides in the CKs group the results were substantially better leading to a total classification accuracy of classifier (using repeated hold-out cross-validation) around $72.1 \%$ in roots and $72.9 \%$ in shoots.

The most identifiable ecotypes were Bay0, Cvi-0, Kon, Ler, RRS_7, Van-0, Ws-0, Ws-2 and they were identified well using both root and shoot data.

When we used only the above ecotypes for classification, the accuracy of the random forest was more than $98 \%$ for the roots which means that these ecotypes differ from the others and among each 
other. In the analysis of shoots, there was a small probability that Ws- 0 and Ler might have some similarities. The confusion matrices for roots and shoots are presented in Tables A3 and A4.

Table A3. Confusion matrix for roots using only the most identifiable ecotypes. The diagonal elements are the correct estimation of the random forest classifier.

\begin{tabular}{cccccccccc}
\hline & \multicolumn{10}{c}{ PREDICTIONS } \\
\hline \multirow{5}{*}{ ACTUAL } & Bay-0 & Cvi-0 & Kon & Ler & RRS7 & Van-0 & Ws-0 & Ws-2 \\
& Bay-0 & 100 & 0 & 0 & 0 & 0 & 0 & 0 & 0 \\
& Cvi-0 & 6 & 92 & 0 & 0 & 2 & 0 & 0 & 0 \\
& Kon & 0 & 0 & 99 & 1 & 0 & 0 & 0 & 0 \\
& Ler & 0 & 0 & 0 & 100 & 0 & 0 & 0 & 0 \\
& RRS7 & 1 & 0 & 0 & 0 & 99 & 0 & 0 & 0 \\
& Van-0 & 0 & 0 & 0 & 0 & 0 & 100 & 0 & 0 \\
& Ws-0 & 0 & 0 & 0 & 0 & 0 & 0 & 100 & 0 \\
Ws-2 & 0 & 0 & 0 & 0 & 0 & 0 & 0 & 100 \\
\hline
\end{tabular}

Table A4. Confusion matrix for shoots using only the most identifiable ecotypes.

\begin{tabular}{cccccccccc}
\hline & & \multicolumn{7}{c}{ PREDICTIONS } \\
\hline & & Bay-0 & Cvi-0 & Kon & Ler & RRS7 & Van-0 & Ws-0 & Ws-2 \\
& Bay-0 & 100 & 0 & 0 & 0 & 0 & 0 & 0 & 0 \\
Cvi-0 & 0 & 97 & 2 & 0 & 0 & 1 & 0 & 0 \\
Kon & 0 & 0 & 100 & 0 & 0 & 0 & 0 & 0 \\
& Ler & 0 & 0 & 0 & 82 & 11 & 0 & 7 & 0 \\
& RRS7 & 0 & 0 & 0 & 0 & 100 & 0 & 0 & 0 \\
& Van-0 & 0 & 0 & 0 & 0 & 0 & 100 & 0 & 0 \\
& Ws_0 & 2 & 2 & 1 & 13 & 0 & 0 & 81 & 1 \\
& Ws_2 & 0 & 0 & 0 & 0 & 0 & 0 & 0 & 100 \\
\hline
\end{tabular}

\section{References}

1. Clark, R.M.; Schweikert, G.; Toomajian, C.; Ossowski, S.; Zeller, G.; Shinn, P.; Warthmann, N.; Hu, T.T.; Fu, G.; Hinds, D.A.; et al. Common sequence polymorphisms shaping genetic diversity in arabidopsis thaliana. Science 2007, 317, 338-342. [CrossRef]

2. Passardi, F.; Dobias, J.; Valério, L.; Guimil, S.; Penel, C.; Dunand, C. Morphological and physiological traits of three major arabidopsis thaliana accessions. J. Plant Physiol. 2007, 164, 980-992. [CrossRef]

3. Kissen, R.; Hyldbakk, E.; Wang, C.W.V.; Sørmo, C.G.; Rossiter, J.T.; Bones, A.M. Ecotype dependent expression and alternative splicing of epithiospecifier protein (ESP) in arabidopsis thaliana. Plant Mol. Biol. 2012, 78, 361-375. [CrossRef]

4. Takahashi, S.; Monda, K.; Negi, J.; Konishi, F.; Ishikawa, S.; Hashimoto-Sugimoto, M.; Goto, N.; Iba, K. Natural variation in stomatal responses to environmental changes among Arabidopsis thaliana ecotypes. PLoS ONE 2015, 10, e0117449. [CrossRef]

5. Clauw, P.; Coppens, F.; De Beuf, K.; Dhondt, S.; Van Daele, T.; Maleux, K.; Storme, V.; Clement, L.; Gonzalez, N.; Inzé, D. Leaf responses to mild drought stress in natural variants of arabidopsis. Plant Physiol. 2015, 167, 800-816. [CrossRef]

6. Clauw, P.; Coppens, F.; Korte, A.; Herman, D.; Slabbinck, B.; Dhondt, S.; Van Daele, T.; De Milde, L.; Vermeersch, M.; Maleux, K.; et al. Leaf growth response to mild drought: Natural variation in arabidopsis sheds light on trait architecture. Plant Cell 2016, 28, 2417-2434. [CrossRef]

7. Verma, V.; Ravindran, P.; Kumar, P.P. Plant hormone-mediated regulation of stress responses. BMC Plant Biol. 2016, 16, 86. [CrossRef] [PubMed]

8. Samsonova, Z.; Kuklova, A.; Mazura, P.; Rotkova, G.; Novak, O.; Brzobohaty, B.; Kiran, N.S. Natural variation in the cytokinin metabolic network in arabidopsis thaliana. Mendelnet 2012, 881-886.

9. Wilkinson, S. Water Use Efficiency and Chemical Signalling. In Water Use Efficiency in Plant Biology; Blackwell Publishing: Bacon, MA, USA, 2004; pp. 75-112. 
10. Zhang, P.; Wang, W.Q.; Zhang, G.L.; Kaminek, M.; Dobrev, P.; Xu, J.; Gruissem, W. Senescence-inducible expression of isopentenyl transferase extends leaf life, increases drought stress resistance and alters cytokinin metabolism in cassava. J. Integr. Plant Biol. 2010, 52, 653-669. [CrossRef] [PubMed]

11. Miyawaki, K.; Matsumoto-Kitano, M.; Kakimoto, T. Expression of cytokinin biosynthetic isopentenyltransferase genes in Arabidopsis: Tissue specificity and regulation by auxin, cytokinin, and nitrate. Plant J. 2004, 37, 128-138. [CrossRef] [PubMed]

12. Takei, K.; Yamaya, T.; Sakakibara, H. Arabidopsis CYP735A1 and CYP735A2 encode cytokinin hydroxylases that catalyse the biosynthesis of trans-Zeatin. J. Biol. Chem. 2004, 279, 41866-41872. [CrossRef] [PubMed]

13. Nordström, A.; Tarkowski, P.; Tarkowska, D.; Norbaek, R.; Åstot, C.; Dolezal, K.; Sandberg, G. Auxin regulation of cytokinin biosynthesis in Arabidopsis thaliana: A factor of potential importance for auxin-cytokinin-regulated development. Proc. Natl. Acad. Sci. USA 2004, 101, 8039-8044. [CrossRef] [PubMed]

14. Schaller, G.E.; Bishopp, A.; Kieber, J.J. The yin-yang of hormones: Cytokinin and auxin interactions in plant development. Plant Cell 2015, 27, 44-63. [CrossRef] [PubMed]

15. Miller, C.O.; Skoog, F.; Okomura, F.S.; Von Saltza, M.H. Isolation, structure and synthesis of kinetin, a substance promoting cell division. J. Am. Chem. Soc. 1956, 75, 1345-1350.

16. Miller, C.O.; Skoog, F.; Von Saltza, M.H.; Strong, F.M. Kinetin, a cell division factor from deoxyribonucleic acid. J. Am. Chem. Soc. 1955, 77, 1392. [CrossRef]

17. Pospíšilová, J.; Haisel, D.; Synková, H.; Bat'ková-Spoustová, P. Improvement of ex vitro transfer of tobacco plantlets by addition of abscisic acid to the last subculture. Biol. Plant. 2009, 53, 617-624. [CrossRef]

18. Kudoyarova, G.; Veselova, S.; Hartung, W.; Farhutdinov, R.; Veselov, D.; Sharipova, G. Involvement of root $\mathrm{ABA}$ and hydraulic conductivity in the control of water relations in wheat plants exposed to increased evaporative demand. Planta 2011, 233, 87-94. [CrossRef]

19. Ghanem, M.E.; Hichri, I.; Smigocki, A.C.; Albacete, A.; Fauconnier, M.L.; Diatloff, E.; Martinez-Andujar, C.; Lutts, S.; Dodd, I.C.; Pérez-Alfocea, F. Root-targeted biotechnology to mediate hormonal signalling and improve crop stress tolerance. Plant Cell Rep. 2011, 30, 807-823. [CrossRef]

20. Wang, T.; Li, C.; Wu, Z.; Jia, Y.; Wang, H.; Sun, S.; Mao, C.; Wang, X. Abscisic acid regulates auxin homeostasis in rice root tips to promote root hair elongation. Front. Plant Sci. 2017, 8, 1121. [CrossRef]

21. Qi, Y. Random Forest for Bioinformatics. In Ensemble Machine Learning: Methods and ApplicatiOns; Springer: Boston, MA, USA, 2012; pp. 307-323. ISBN 9781441993267.

22. Gelová, Z.; Ten Hoopen, P.; Novák, O.; Motyka, V.; Pernisová, M.; Dabravolski, S.; Didi, V.; Tillack, I.; OklešA Ková, J.; Strnad, M.; et al. Antibody-mediated modulation of cytokinins in tobacco: Organ-specific changes in cytokinin homeostasis. J. Exp. Bot. 2018, 69, 441-454. [CrossRef]

23. Bishopp, A.; Lehesranta, S.; Vatén, A.; Help, H.; El-Showk, S.; Scheres, B.; Helariutta, K.; Mähönen, A.P.; Sakakibara, H.; Helariutta, Y. Phloem-transported cytokinin regulates polar auxin transport and maintains vascular pattern in the root meristem. Curr. Biol. 2011, 21, 927-932. [CrossRef] [PubMed]

24. Corbesier, L.; Prinsen, E.; Jacqmard, A.; Lejeune, P.; Van Onckelen, H.; Périlleux, C.; Bernier, G. Cytokinin levels in leaves, leaf exudate and shoot apical meristem of Arabidopsis thaliana during floral transition. J. Exp. Bot. 2003, 54, 2511-2517. [CrossRef]

25. Hirose, N.; Takei, K.; Kuroha, T.; Kamada-Nobusada, T.; Hayashi, H.; Sakakibara, H. Regulation of cytokinin biosynthesis, compartmentalization and translocation. J. Exp. Bot. 2008, 59, 75-83. [CrossRef] [PubMed]

26. Matsumoto-Kitano, M.; Kusumoto, T.; Tarkowski, P.; Kinoshita-Tsujimura, K.; Václavíková, K.; Miyawaki, K.; Kakimoto, T. Cytokinins are central regulators of cambial activity. Proc. Natl. Acad. Sci. USA 2008, 105, 20027-20031. [CrossRef] [PubMed]

27. Kudo, T.; Kiba, T.; Sakakibara, H. Metabolism and long-distance translocation of cytokinins. J. Integr. Plant Biol. 2010, 52, 53-60. [CrossRef]

28. James, G.; Witten, D.; Hastie, T.; Tibshirani, R. An Introduction to Statistical Learning; Springer: New York, NY, USA, 2013; Volume 6, ISBN 9780387781884.

29. Janečková, H.; Husičková, A.; Ferretti, U.; Prčina, M.; Pilařová, E.; Plačková, L.; Pospíšil, P.; Doležal, K.; Špundová, M. The interplay between cytokinins and light during senescence in detached arabidopsis leaves. Plant Cell Environ. 2018, 41, 1870-1885. [CrossRef] 
30. Jin, S.H.; Ma, X.M.; Kojima, M.; Sakakibara, H.; Wang, Y.W.; Hou, B.K. Overexpression of glucosyltransferase UGT85A1 influences trans-zeatin homeostasis and trans-zeatin responses likely through O-glucosylation. Planta 2013, 237, 991-999. [CrossRef]

31. Hou, B.; Lim, E.K.; Higgins, G.S.; Bowles, D.J. N-glucosylation of cytokinins by glycosyltransferases of arabidopsis thaliana. J. Biol. Chem. 2004, 279, 47822-47832. [CrossRef]

32. Wang, J.; Ma, X.M.; Kojima, M.; Sakakibara, H.; Hou, B.K. Glucosyltransferase UGT76C1 finely modulates cytokinin responses via cytokinin $\mathrm{N}$-glucosylation in arabidopsis thaliana. Plant Physiol. Biochem. 2013, 65, 9-16. [CrossRef]

33. Brzobohatý, B.; Moore, I.; Kristoffersen, P.; Bako, L.; Campos, N.; Schell, J.; Palme, K. Release of active cytokinin by a beta-glucosidase localized to the maize root meristem. Science 1993, 262,1051-1054. [CrossRef]

34. Pernisová, M.; Klíma, P.; Horák, J.; Válková, M.; Malbeck, J.; Souček, P.; Reichman, P.; Hoyerová, K.; Dubová, J.; Friml, J.; et al. Cytokinins modulate auxin-induced organogenesis in plants via regulation of the auxin efflux. Proc. Natl. Acad. Sci. USA 2009, 106, 3609-3614. [CrossRef] [PubMed]

35. Li, P.; Lei, K.; Li, Y.; He, X.; Wang, S.; Liu, R.; Ji, L.; Hou, B. Identification and characterization of the first cytokinin glycosyltransferase from rice. Rice 2019, 12, 19. [CrossRef] [PubMed]

36. Bartrina, I.; Otto, E.; Strnad, M.; Werner, T.; Schmülling, T. Cytokinin regulates the activity of reproductive meristems, flower organ size, ovule formation, and thus seed yield in Arabidopsis thaliana. Plant Cell 2011, 23, 69-80. [CrossRef] [PubMed]

37. Ramireddy, E.; Hosseini, S.A.; Eggert, K.; Gillandt, S.; Gnad, H.; von Wirén, N.; Schmülling, T. Root engineering in barley: Increasing cytokinin degradation produces a larger root system, mineral enrichment in the shoot and improved drought tolerance. Plant Physiol. 2018, 177, 1078-1095. [CrossRef] [PubMed]

38. Skalák, J.; Vercruyssen, L.; Claeys, H.; Hradilová, J.; Černý, M.; Novák, O.; Plačková, L.; Saiz-Fernández, I.; Skaláková, P.; Coppens, F.; et al. Multifaceted activity of cytokinin in leaf development shapes its size and structure in arabidopsis. Plant J. 2019, 97, 805-824. [CrossRef] [PubMed]

39. Werner, T.; Holst, K.; Pörs, Y.; Guivarc'h, A.; Mustroph, A.; Chriqui, D.; Grimm, B.; Schmülling, T. Cytokinin deficiency causes distinct changes of sink and source parameters in tobacco shoots and roots. J. Exp. Bot. 2008, 59, 2659-2672. [CrossRef]

40. Skalák, J.; Cerný, M.; Jedelský, P.; Dobrá, J.; Ge, E.; Novák, J.; Hronková, M.; Dobrev, P.; Vanková, R.; Brzobohatý, B. Stimulation of ipt overexpression as a tool to elucidate the role of cytokinins in high temperature responses of arabidopsis thaliana. J. Exp. Bot. 2016, 67, 2861-2873. [CrossRef]

41. Wilkinson, S.; Kudoyarova, G.R.; Veselov, D.S.; Arkhipova, T.N.; Davies, W.J. Plant hormone interactions: Innovative targets for crop breeding and management. J. Exp. Bot. 2012, 63, 3499-3509. [CrossRef]

42. Chapin, F.S. Integrated responses of plants to stress. Bioscience 1991, 41, 29-36. [CrossRef]

43. Dobrev, P.I.; Kamínek, M. Fast and efficient separation of cytokinins from auxin and abscisic acid and their purification using mixed-mode solid-phase extraction. J. Chromatogr. A 2002, 950, 21-29. [CrossRef]

44. Svačinová, J.; Novák, O.; Plačková, L.; Lenobel, R.; Holík, J.; Strnad, M.; Doležal, K. A new approach for cytokinin isolation from arabidopsis tissues using miniaturized purification: Pipette tip solid-phase extraction. Plant Methods 2012, 8, 17. [CrossRef] [PubMed]

45. Floková, K.; Tarkowská, D.; Miersch, O.; Strnad, M.; Wasternack, C.; Novák, O. UHPLC-MS/MS based target profiling of stress-induced phytohormones. Phytochemistry 2014, 105, 147-157. [CrossRef] [PubMed]

46. de Winter, J.C.F. Using the student's t-test with extremely small sample sizes. Pract. Assess. Res. Eval. 2013, 18, 2.

47. Ruxton, G.D. The unequal variance t-test is an underused alternative to Student's t-test and the mann-whitney U test. Behav. Ecol. 2006, 17, 688-690. [CrossRef]

48. Breiman, L. Random forests. Mach. Learn. 2001, 45, 5-32. [CrossRef]

49. Team RStudio. RStudio: Integrated Development Environment for R; RStudio Inc.: Boston, MA, USA, 2015; Available online: http://www.rstudio.com (accessed on 17 September 2019).

50. El-Soda, M.; Kruijer, W.; Malosetti, M.; Koornneef, M.; Aarts, M.G.M. Quantitative trait loci and candidate genes underlying genotype by environment interaction in the response of arabidopsis thaliana to drought. Plant Cell Environ. 2015, 38, 585-599. [CrossRef]

51. Hothorn, M.; Dabi, T.; Chory, J. Structural basis for cytokinin recognition by arabidopsis thaliana histidine kinase 4. Nat. Chem. Biol. 2011, 7, 766-768. [CrossRef] 
52. Bartrina, I.; Jensen, H.; Novák, O.; Strnad, M.; Werner, T.; Schmülling, T. Gain-of-function mutants of the cytokinin receptors AHK2 and AHK3 regulate plant organ size, flowering time and plant longevity. Plant Physiol. 2017, 173, 1783-1797. [CrossRef]

53. Miwa, K.; Ishikawa, K.; Terada, K.; Yamada, H.; Suzuki, T.; Yamashino, T.; Mizuno, T. Identification of amino acid substitutions that render the arabidopsis cytokinin receptor histidine kinase AHK4 constitutively active. Plant Cell Physiol. 2007, 48, 1809-1814. [CrossRef]

54. Yamada, H.; Suzuki, T.; Terada, K.; Takei, K.; Ishikawa, K.; Miwa, K.; Yamashino, T.; Mizuno, T. The arabidopsis AHK4 histidine kinase is a cytokinin-binding receptor that transduces cytokinin signals across the membrane. Plant Cell Physiol. 2001, 42, 1017-1023. [CrossRef]

55. Hošek, P.; Hoyerová, K.; Kiran, N.S.; Dobrev, P.I.; Zahajská, L.; Filepová, R.; Motyka, V.; Müller, K.; Kamínek, M. Distinct metabolism of $N$-glucosides of isopentenyladenine and trans-zeatin determines cytokinin metabolic spectrum in arabidopsis. New Phytol. 2019. [CrossRef] [PubMed]

(C) 2020 by the authors. Licensee MDPI, Basel, Switzerland. This article is an open access article distributed under the terms and conditions of the Creative Commons Attribution (CC BY) license (http://creativecommons.org/licenses/by/4.0/). 